Akute Diarrhö

\section{Probiotika ermöglichen kausale Therapie}

- Probiotika werden zunehmend als kausale Therapiemöglichkeit bei akutem Durchfall eingesetzt. Hoch dosiert zugeführte Bakterien wie Lactobazillen bilden einen Biofilm auf den Schleimhautzellen des Darms, der die Adhäsion pathogener Keime verhindert, und stimulieren das gastrointestinale Immunsystem. Das natürliche Gleichgewicht einer gesunden Darmflora wird wiederhergestellt, erklärte Priv.Doz. Dr. Harald Matthes, Berlin.

Mit am besten sei die Studienlage für Lactobazillen. Die Wirsamkeit von Lacteol ${ }^{\circledR}$, in dem L. fermentum und L. delbrueckii hoch dosiert und gefriergetrocknet vorliegen, sodass keine Kühlung erforderlich ist, wurde in zwei randomisierten kontrollierten Studien belegt. In der einen Studie bei Erwachsenen wurde die Durchfalldauer nach Therapiebeginn um fast 50\% (um

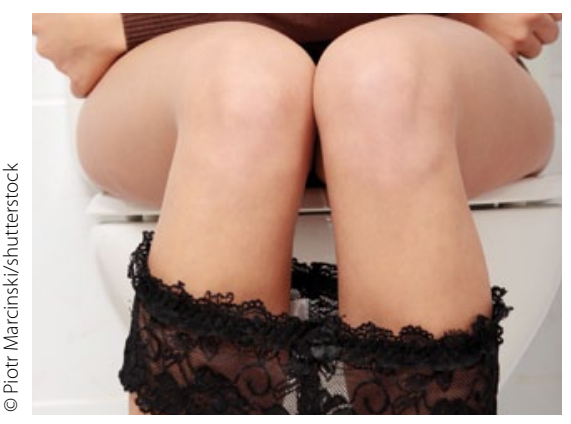

0,7-1,4 Tage), in der anderen bei Kindern um 73\% (Symptomdauer nur 8,2 Std. vs. 30,4 Std. bei Placebo) verkürzt (J Pediatr Gastroenterol Nutr 2007; 44: 571-576).

Lactobazillen sind bei viraler wie auch bakterieller Genese des Durchfalls und bei antibiotikaassoziierten Diarrhöen gleichermaßen wirksam und zur Durchfalltherapie gut geeignet, betonte Matthes. Motilitätshemmer sind bei akuten Durchfällen zwar sehr rasch wirksam, haben aber nach Ansicht des Gastroenterologen nur eine soziale Indikation, etwa auf Reisen oder beim Konzert, da auch die Ausscheidung pathogener Keime verlangsamt wird.

- Roland Fath

Quelle: Lacteol ${ }^{\oplus}$-Presseworkshop „Die unterschätzte Gefahr: Durchfall“, Hamburg, August 2012 (Veranstalter: Pohl-Boskamp)

Lebensqualität bei pulmonaler Hypertonie Ähnlich beeinträchtigt wie bei
fortgeschrittenem Krebsleiden

- Patienten mit pulmonaler Hypertonie $(\mathrm{PAH})$ leiden oft nicht nur unter ihrer Grunderkrankung, sondern zusätzlich unter Depression, Stress, Angst- und Schlafstörungen, die sich alle weiter negativ auf das Befinden auswirken. Eine PAH hat somit eine vergleichbare Auswirkung auf die Lebensqualität wie metastasierte Krebsleiden oder eine Querschnittslähmung, erklärte Prof. Marion Delcroix, Leuven/Belgien. Sie unterstrich den Stellenwert der Erfassung der Lebensqualität z. B. mithilfe von SF-36, CAMPHOR bzw. MLHFQ. Wichtig sei gerade die subjektive Beurteilung durch die Betroffenen selbst, die erheblich von der äußeren Einschätzung divergieren kann.

Delcroix erklärte außerdem, wie die Lebensqualität von PAH-Patienten optimiert werden kann. Untersuchungen zeigten den Nutzen einer frühen palliativen Versorgung, betonte Delcroix und plädierte für eine vorgezogene Palliativversorgung, die ihrer Meinung nach unmittelbar nach der Diagnosestellung beginnen sollte.

Delcroix zeigte zudem die überzeugenden Ergebnisse der SUPER-1-Studie zur Verbesserung der Lebensqualität bei PAH, gemessen anhand des SF-36. Dabei hatten verschiedene Dosierungen von Sildenafil $\left(\right.$ Revatio $\left.^{\oplus}\right)$ im Placebovergleich einen positiven Einfluss auf Parameter wie Funktionstüchtigkeit, allgemeiner Gesundheitszustand und körperliche Schmerzen.

\footnotetext{
- Dr. Yvette C. Zwick

Quelle: Symposium „Optimal management of pulmonary arterial hypertension patients: Are we there yet? A case-based approach", ESC-Kongress,
} München, August 2012 (Veranstalter: Pfizer)

Patienten mit Colitis ulcerosa aufklären Weniger Rückfälle durch bessere
Therapietreue

- Bei der Colitis ulcerosa (CU) bedarf es zum Erhalt der Remission und zur Karzinomprophylaxe einer langfristigen Dauertherapie mit oral oder topisch-rektal gegebenen Aminosalicylaten (Mesalazin, 5-ASA), erklärte Prof. Ingolf Schiefke, Leipzig. Dies dem Patienten begreiflich zu machen, sei nicht immer einfach. Untersuchungen zeigen, dass unter einer Langzeitbehandlung (mehr als elf Jahre) nur noch $65 \%$ aller Patienten die Therapie wie verordnet anwenden. Die mangelnde Therapietreue werde mit einem bis zu fünffach höheren Rückfallrisiko bestraft. Gründe können zeitaufwendige rektale Anwendungen von Klysmen oder Schäumen sein, die sich nicht gut in den Alltag der meist noch berufstätigen Patienten integrieren lassen.

Einfacher anzuwenden ist Mesalazin in oraler Form, etwa MMX ${ }^{\circledR}$-Mesalazin (Meza- vant ${ }^{\oplus}$ ). Wie Schiefke erläuterte, sorgt die Multimatrix-Formulierung für eine Freisetzung erst im Kolon und für eine ausreichend lange Wirkdauer, die eine tägliche Einmalgabe ermöglicht.

Eine aktuelle Studie weist darauf hin, dass die Einmalgabe von 2,4 $\mathrm{g} \mathrm{MMX}^{\circledR}$ Mesalazin die Remissionserhaltung inklusive mukosaler Heilung bei über $83 \%$ der CU-Patienten ermöglicht (D'Haens $\mathrm{G}$ et al. Am J Gastroenterol 2012, 107(7): 10641077). Mesalazin in der Multimatrix-Formulierung kann in der langfristigen CU-Behandlung die Therapietreue deutlich unterstützen, so Schiefke.

\footnotetext{
- Dagmar Jäger-Becker

Quelle: Satellitensymposium „Remissionserhalt der Colitis ulcerosa: Wer erwartet was?", DGVS-Jahrestagung, Hamburg, September 2012 (Veranstalter: Shire Deutschland)
} 